\title{
Chapter
}

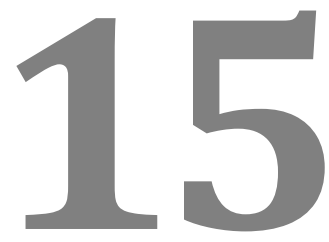

\section{IMMUNOMODULATION BY ANTIBIOTICS}

\section{Leonard Blum ${ }^{1}$, Susanne Schiffmann ${ }^{1,2}$, and Michael J. Parnham ${ }^{2,3 *}$}

${ }^{1}$ Pharmazentrum Frankfurt/ZAFES, Institute for Clinical Pharmacology, Goethe University Hospital Frankfurt, Theodor-Stern-Kai 7, 60590 Frankfurt/Main, Germany

${ }^{2}$ Fraunhofer Institute for Molecular Biology and Applied Ecology IME, Branch for Translational Medicine and Pharmacology TMP, Theodor-Stern-Kai 7, 60596 Frankfurt am Main, Germany

${ }^{3}$ Pharmacological Institute for Life Scientists, Goethe University Frankfurt, Max-von-Laue-Str. 9, 60438 Frankfurt

*Email: michael.parnham@ime.fraunhofer.de 


\section{Contents}

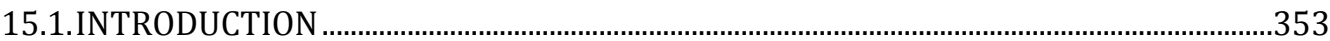

15.2. THE INFLAMMATORY PROCESS AND INTERACTIONS WITH

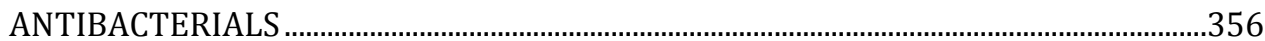

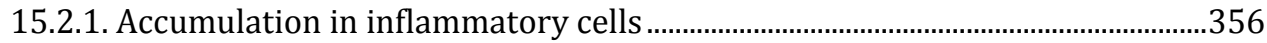

15.2.2. Effects on immune cell infiltration and vasodilation............................................357

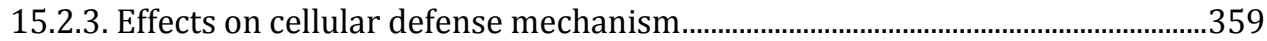

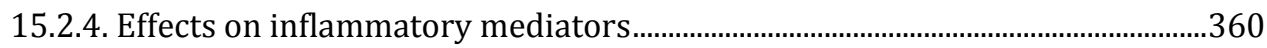

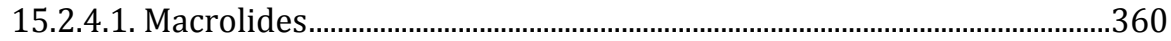

15.2.4.2. Quinolones/Fluoroquinolones ...............................................................361

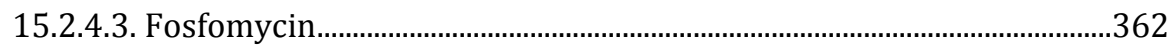

15.2.4.4. Cyclines/Tetracyclines .............................................................................. 362

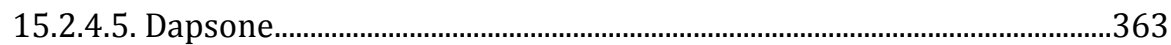

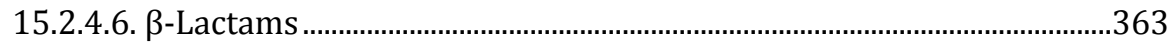

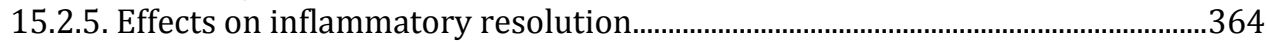

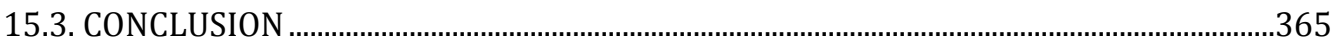

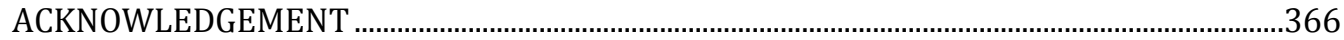

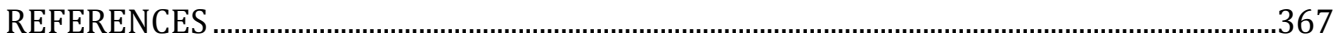




\subsection{INTRODUCTION}

Since more and more bacteria are developing resistance against the direct impact of antibiotics, it is important to consider the broader effects of these drugs for strategies to combat resistance. Several antibacterials are able to exert indirect antibacterial actions that can be utilized for this purpose. The modulation of the immune system, as part of the host defense response, can boost antibacterial defense and facilitate clearance of bacteria [1]. In this connection, immunomodulation by several antibiotics, particularly macrolides but also quinolones, can either promote bacterial killing or facilitate resolution of inflammation and reduce bystander tissue injury. Some of these antibiotics can modify bacterial inflammation even when resistance to the primary effects of the drugs occur. In some cases, these antibiotics are even effective against respiratory viral infections by reducing the cytokine storm [2]. Incorporation of immunomodulation into the activity profile of antibiotics can thus, expand their breadth of activity in bacterial infections, independent of the degree of bacterial resistance [3].

Most infections are accompanied by inflammation which is characterized by increased accumulation of inflammatory cells (monocytes, macrophages, granulocytes, plasma cells, lymphocytes and platelets). Together with the tissue endothelial cells and fibroblasts, these cells release a variety of enzymes, cytokines, growth factors and lipid mediators which can lead to tissue damage. Tissue injury is dependent on the type of immune response. For example, neutrophil-dominated inflammatory diseases that include bacterial infections as well as active phases of rheumatoid arthritis, cystic fibrosis, bronchitis and chronic obstructive pulmonary disease, are characterized by the release, among other products, of proteinases and reactive oxygen species (ROS) by neutrophils which also exert antibacterial responses [4].

Neutrophils are an essential component of the host defense system against bacterial infections. It has become clear that the modulation of neutrophil activation is a promising approach for the regulation of inflammatory conditions [4]. In their role as a decisive defense force, neutrophils have developed several mechanisms of antibacterial activities. First of all, the phagocytically triggered respiratory burst is an effective way of destroying invading microorganisms. Thereby, a huge quantity of toxic ROS (e.g. superoxide anion, singlet oxygen, hydroxyl radical, hydrogen peroxide, hypohalous acid) are generated. Secondly, neutrophils generate secretory granules which contain effective degradative enzymes and bactericidal proteins (e.g. myeloperoxidase, elastase, cathepsin, collagenase, neuraminidase, heparanase, defensins). There is a risk, however, that these defensive mechanisms might not only kill the invading bacteria, but also harm the host tissue. Consequently, resolution of this acute inflammatory response is 
essential to avoid unintended tissue damage [5]. Locally released lipids like prostaglandin D2 derivatives exert an important role in the resolution of the inflammatory state by promoting apoptosis in neutrophils [6]. The programmed cell death of neutrophils provokes reduced release of proinflammatory cytokines $[7,8]$. During the progress of inflammatory resolution, macrophages play a crucial role through their phagocytosis of apoptotic neutrophils. Furthermore, macrophages support the tissue healing process by releasing growth factors, removing tissue debris and stimulating formation of replacement connective tissue [8]. The balance between the initiation of inflammation which results in the killing of the pathogens and the well-timed resolution of the inflammation to prevent tissue damage is a complex mechanism which can be modulated by several antibacterials [1,3]. The immunomodulating effects of antibacterials can either promote the initial antibacterial defense and/or the subsequent process of inflammatory resolution. Therefore, an important factor in the pharmacological modulation of inflammation is the timing of the treatment. Stimulation of the acute inflammatory response, e.g. by additional activation of neutrophils, promotes the removal of the bacteria and boosts thereby, the host defense. At a later time point, the stimulation of leukocyte apoptosis and the reduction of released proinflammatory cytokines is important to prevent tissue damage in infectious diseases and non-infectious chronic inflammatory conditions. Especially in patients with chronic inflammatory disorders, the anti-inflammatory properties of antibacterials and other drugs are implemented to limit ongoing tissue damage (Figure 1).

The particular benefits of these immunomodulatory effects include promotion of host defense, even when bacterial resistance may dampen sensitivity to the direct impact of the antibiotics. The relevant phases of the inflammatory process and the opportunity to modulating these phases with antibacterial agents will be described in the following section. 


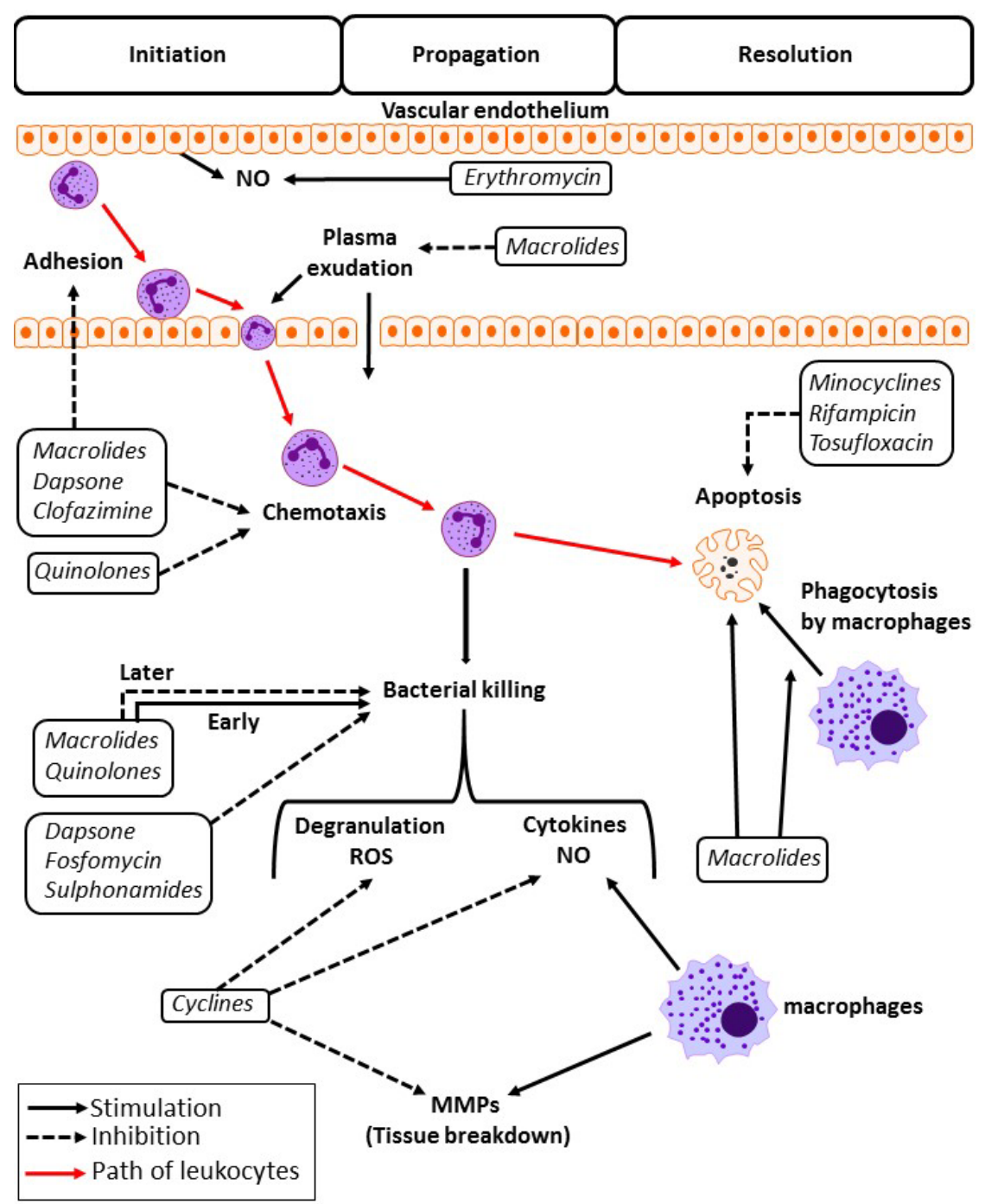

Figure 1. Overview of the main actions of antibiotics in the three main phases of inflammation. Stimulatory actions are indicated by unbroken lines while inhibitory actions are denoted in dashed lines. Antibiotics are indicated by italic letters. The initiation phase consists of vasodilation, adhesion, chemotaxis, transendothelial migration of leukocytes and plasma exudation. Several antibiotics show inhibitory actions during this phase while erythromycin has stimulatory effects on endothelial cells and NO production. During propagation, the inflammatory leukocytes are activated and inflammatory mediators, as well as degradative enzymes, are released. This leads to the destruction of the invading pathogens. Plasma exudation is further promoted and tissue damage near the inflammation side occurs. In this phase, antibiotics have mainly inhibitory effects. Macrolides (azithromycin) and quinolones (minocycline) exhibit stimulatory actions in the initiation and the early phase of propagation. Inflammatory resolution is associated with enhanced apoptosis and the release of anti-inflammatory cytokines. Apoptosis is promoted by macrolides but inhibited by several other antibiotics. MMP = metalloproteinase, $\mathrm{NO}=$ nitric oxid. 


\subsection{THE INFLAMMATORY PROCESS AND INTERACTIONS WITH ANTIBACTERIALS}

Several antibacterials show inflammation modulating effects on leukocytes, especially on neutrophils. Among others, macrolides, ketolides, quinolones and tetracyclines show the most pronounced effects and are reviewed here with regard to their immunomodulatory properties

\subsubsection{Accumulation in inflammatory cells}

To be able to exert immunomodulating effects, antibacterials must first accumulate in the immune cells. There is evidence that macrolides reach concentrations of up to several hundred-fold higher in inflammatory cells in comparison to the extracellular fluid $[4,9]$. This results in effective delivery of the antibacterials to the site of inflammation/infection. The exact mechanism by which this intracellular accumulation of macrolides is achieved is yet unclear, but there are hints that it is an active protein-mediated process [10]. Accumulation takes place particularly in the cytoplasm and the azurophilic granules of neutrophils, resulting in effective delivery of the macrolides to phagocytosed bacteria. Cytokines enhance macrolide accumulation in macrophages in vitro, so that even more pronounced accumulation of macrolides at the site of inflammation/infection might be observed under inflammatory conditions in vivo [11].

There are several differences with respect to the efflux of the various macrolides. Clarithromycin and erythromycin show a very fast release from leukocytes while azithromycin remains longer within the cells $[9,12,13]$. The prolonged residence of azithromycin facilitates a longer antibacterial effect and a pronounced immunomodulating effect which might not be observed in short-term cell culture experiments in vitro.

Macrolides show the most distinct accumulation in immune cells, but other antibacterials also show similar effects. For example, clindamycin shows an approximate 20 -fold intracellular accumulation within alveolar macrophages via the nucleoside transport system [14]. Chloramphenicol, lincomycin, tetracycline and rifampin also show selective accumulation by about 2- to 5fold. Within neutrophils, the concentrations of ciprofloxacin and quinolones are about 5-fold higher than in the extracellular fluid [15]. After accumulation in the immune cells, the antibacterials can modulate the further steps of the immune response. 


\subsubsection{Effects on immune cell infiltration and vasodilation}

One of the first essential steps in the inflammatory process is the infiltration of neutrophils and further immune cells into the tissue. This recruitment includes a sequence of events which involves the specific arrest of the immune cells on the vascular endothelium and their transmigration across the endothelial cell barrier (Figure 1). The adhesion process is characterized by four phases (margination, capture, rolling and adhesion) which are mediated by several cell adhesion molecules of the integrin and selectin family. The expression of these adhesion molecules is stimulated by pro-inflammatory cytokines [16]. Furthermore, chemokines and complement anaphylatoxins influence the directed migration of the immune cells. Vasodilatory factors such as prostaglandin $\mathrm{E}_{2}$ lead to plasma exudation and swelling which also affect the infiltration process. These initial steps in the process of inflammatory response are modified by several antibacterials $[1,3,17]$.

An important molecule for the adhesion process is Mac-1 (CD11b/CD18). It has been shown in patients with diffuse panbronchiolitis (DPB) that Mac-1 is upregulated on peripheral neutrophils compared to healthy subjects [18]. The number of infiltrating immune cells at the site of inflammation is reduced by inhibition of these adhesion molecules. Several macrolides have an impact on the adhesion molecules of neutrophils. Roxithromycin is able to reduce the expression of Mac-1 on peripheral neutrophils from patient with DPB. Treatment for 2 weeks with erythromycin inhibits expression of Mac-1 and L-selectin which results in a reduced infiltration of macrophages and neutrophils into the site of inflammation in rats with experimental otitis media [19]. Additionally, erythromycin reduced L-selectin expression in an LPSinduced neutrophil recruiting model in rats [20]. Both roxithromycin and erythromycin decrease intercellular adhesion molecule-1 (ICAM-1) expression, which is a surface protein of the integrin family [21,22]. Roxithromycin was ineffective on whole blood cells in experiments in vitro, while a reduction of Mac-1 expression on neutrophils was observed after treatment of patients with lower respiratory tract disease like DPB [18]. This indicates that prolonged treatment is necessary for the inhibitory effects. That delayed action has the advantage that at the beginning of infection and of treatment, when the immune response is needed, the macrolides did not reduce the infiltration of immune cells. Later on, when the immune response switches to resolution, the macrolides promote the process by preventing further immune cell migration into the tissue. Furthermore, roxithromycin was able to inhibit adhesion of neutrophils to epithelial cells in vitro [21]. Clarithromycin reduced the expression of several adhesion molecules, like vascular cell adhesion molecule1 (VCAM-1), lymphocyte function associated antigen-3 (LFA-3) and ICAM-1 in human bronchial epithelial and synovial cells [23]. Taken together, several studies showed an inhibitors effect of macrolides on adhesion molecule expression and thereby, they reduce neutrophil migration into the tissue. 
Dapsone is also able to inhibition the expression of cell adhesion molecules [24].

The generation of nitric oxide (NO) is also influenced by some macrolides. NO is an important second messenger in the mechanism of ROS regulation within the inflammatory response. It is produced by the NO synthases (NOS) in the first stages of the inflammatory response and can either enhance vasodilation or influence the migration properties of leukocytes.

It has been reported that erythromycin stimulates endothelial NOS (eNOS) production in a protein kinase A-dependent mechanism [25]. This results in inhibition of leukocyte adhesion to endothelial cells and thereby, migration into the tissue. However, there are contradictory reports on suppression of the inducible NOS (iNOS) in pulmonary alveolar macrophages by erythromycin [26]. The results indicate that macrolides can reduce or increase NO synthesis probably depending on the type of NOS with which they interact. Not only macrolides are able to influence the adhesion and migration steps of leukocytes. Clofazimine shows beneficial effects in several skin diseases which might be based on the inhibition of ICAM-1 and HLA-DR [27].

The next important step during the migration process is the attraction of the immune cells by chemokines. A concentration gradient leads the immune cells towards the site of inflammation. The influence of macrolides on neutrophilic chemotaxis is controversial. Several reports showed that long-term treatment with erythromycin reduced the neutrophil chemotactic activity in DPB $[28,29]$. In different acute lung injury models in mice, erythromycin also showed an inhibiting effect on neutrophil migration [28,30,31]. But macrolides did not show effect on neutrophil chemotaxis in every studies. For example, in vitro models showed that a much higher dose of macrolides than the therapeutic dose was needed to inhibit chemotaxis of isolated peripheral blood neutrophils from healthy volunteers [32]. Some studies even report an enhancing effect of erythromycin on neutrophil migration in vitro. It is difficult to distinguish whether the observed effects are due to reduced chemotaxis or due to reduced production of chemoattractants and/or a decreased expression of adhesion molecules [33,34].

Quinolones also reduce rat macrophage chemotaxis. The effects are concentration-dependent and significant yet they do not provide a promising approach since the effects are not strong enough for clinical application [35]. It has been reported that clofazimine shows inhibitory effects on neutrophil motility ex vivo [36].

The assumption that macrolides inhibit plasma exudation and cell infiltration in vivo is also supported by reports showing the efficacy of these antibacterials in the model of carrageenan-induced paw oedema, which is a standard animal model for the investigation of anti-inflammatory compounds [37]. Pre-treatment with erythromycin had protective effects on inflammatory reactions of the airways in rats after E. coli lipopolysaccharide (LPS)-induction 
[38]. These effects seemed to be dependent on neutrophils since there was no protective effect visible in neutropenic rats. This hypothesis is supported by another study which showed that erythromycin and clarithromycin inhibit LPS-induced neutrophil recruitment in the trachea of guinea pigs [39]. In a rat model for lung injury erythromycin and josamycin showed a reduction of NO concentrations in exhaled air and an inhibition of neutrophil accumulation [26].

\subsubsection{Effects on cellular defense mechanism}

After the migration process, the immune cells reach the site of infection where they tackle the invading pathogens. Stimulation of the leukocytes is an essential step in the process at this stage. Neutrophilic granules release a broad range of enzymes, like lysozyme, which directly attack the bacteria. Opsonization of the micro-organisms by immunoglobulins and the complement system enables phagocytosis of the bacteria and the ensuing oxidative burst, which comprises the generation of massive amounts of ROS to destroy the cellular components of the invading pathogens. This process is stimulated by numerous chemokines which further activate the cells and also stimulate other inflammatory mechanisms. The degranulation of neutrophils is directly affected by macrolides in vitro [35]. Additionally, macrolides, with the exception of roxithromycin, stimulate macrophage phagocytosis, chemotaxis and cytocidal activity against the yeast Candida albicans [40]. These host defense promoting effects of macrolides can also be seen in animal models. The administration of erythromycin or roxithromycin $\left(10 \mathrm{mg} \mathrm{kg}^{-1}\right)$ for 28-days promotes pro-inflammatory cytokine production by isolated macrophages and interleukin-2 (IL-2) production by isolated splenocytes in healthy mice. Interestingly, these effects did not occur after a 7-day treatment [41,42]. Furthermore, 14-day roxithromycin treatment in healthy guinea pigs increased the oxidative burst capacity of neutrophils [43]. But not only macrolides are able to boost host defense mechanisms. Quinolones, $\beta$-lactams and cephalosporins also promote neutrophil bacterial killing partly by enhancing phagocytosis and oxidative burst capacity in vitro [35]. Nevertheless, granulocyte functions are not affected by quinolones at clinically relevant concentrations [44]. The majority of quinolones, especially ciprofloxacin, influence pro-inflammatory cytokine gene expression like IL-2 and interferon gamma (IFN- $\gamma$ ), by activation of the nuclear factor AP-1, probably accounting for their role as immunomodulators [44]. Studies on the actions of the inflammagen zymogen A from $S$. aureus showed that stimulation of THP-1 monocytic cells in vitro by moxifloxacin, a fluoroquinolone, is biphasic [45]. At the start, moxifloxacin enhances the release of hydrogen peroxide and NO, but after 4 hours inhibition of pro-inflammatory cytokines, lipid peroxidation and lysosomal enzyme release occurs. This is an interesting property which could be used for immunomodulatory therapy: The enhancement of the proinflammatory processes supports the killing of the invading pathogens while 
the anti-inflammatory effects in the second phase enable inflammatory resolution and tissue regeneration. Such biphasic activities have also been reported for azithromycin in studies on healthy humans treated for three consecutive days $\left(500 \mathrm{mg} \mathrm{d}^{-1}\right)$ [46]. Initially, azithromycin increased the neutrophil degranulating effects but 2,5-24h after the last dose, the azurophilic granule enzyme activities in the cells decreased while there was a corresponding increase in the serum. Ex vivo experiments showed that the oxidative response to particulate stimulus of opsonized zymosan was increased. High concentrations of azithromycin were reported in the serum and in neutrophils. IL-6 and chemokine concentrations in the serum were reduced within the non-pathological range and the oxidative burst capacity was downregulated while the apoptosis of neutrophils was enhanced up to 28 days after the last administered dose. These biphasic effects shown by azithromycin and probably other antibacterials like quinolones, provide a mechanism for an enhanced defense mechanism of the host against invading pathogens and an improved inflammatory resolution to prevent tissue damage through prolonged inflammation.

\subsubsection{Effects on inflammatory mediators}

Several antibacterials show anti-inflammatory effects through inhibition of pro-inflammatory mediators, cytokines and ROS. These antibacterials include macrolides, quinolones and tetracyclines. The anti-inflammatory effects can be made use of due enhance the resolution of inflammation after the successful elimination of the invading pathogens. The most important anti-inflammatory effects of selected antibiotics will be introduced in this chapter.

\subsubsection{Macrolides}

Macrolides have widespread effects on the immune response as partly addressed in the previous chapters. They inhibit the secretion of proinflammatory cytokines, ROS and other pro-inflammatory mediators in vitro while anti-inflammatory cytokines are also modified in multiple ways [47-49]. The modulation of ROS generation has several immunomodulatory consequences since ROS exert miscellaneous physiological effects. A major consequence of ROS action is cell and tissue damage but ROS are also needed for several physiological cellular signal pathways and regulation processes [50]. Macrolides reduce the production of ROS in neutrophils [51]. As a possible mechanism, it has been proposed that macrolides enhance the membrane-destabilizing effect of bioactive phospholipids (e.g. lysophosphatidylcholine, lyso-PAF and platelet-activation factor) and thereby inhibit superoxide production [52]. Erythromycin, clarithromycin and roxithromycin pretreatment of rats with acute carrageenan-induced pleurisy resulted in a reduction of prostaglandin $\mathrm{E}_{2}$, TNF- $\alpha$ levels and NO production [33]. These three macrolides also reduced NO, IL-1 $\beta$, IL- 6 and TNF- $\alpha$ plasma levels as well as lung iNOS mRNA level after i.p. injection of LPS into mice. 
Interestingly, josamycin did not show these effects [53]. In a similar in vivo experiment with i.p. LPS injections, several modified macrolides were tested. These derivatives do not show antibacterial effects but still are able to reduce cytokine production and neutrophilia in vitro [54]. These findings indicate that the direct antibacterial effects and immunomodulation are partially separable properties and more anti-inflammatory substances can probably be developed based on the structure of macrolides. In general, the anti-inflammatory mechanism of macrolides seems to be rather slow. Studies in a peritonitis model induced by zymosan showed that the anti-inflammatory effects of erythromycin achieve a peak 28 days after (pre-) treatment $[55,56]$. Roxithromycin also showed a time-dependency, giving the most pronounced reduction of the cytokine secretion (IL- $1 \beta$, TNF- $\alpha$ ) in a mouse endotoxin LPSinduced inflammation, after seven weeks treatment [57]. These long-term effects underlie the use of macrolides in chronic inflammatory diseases. Investigations of the effects of erythromycin and azithromycin in an adjuvantinduced arthritis model in rats showed that anti-inflammatory effects were exerted through a reduction in circulating lysosomal enzyme activities [58]. Furthermore, the prominent transcription factors NF- $\mathrm{KB}$ and AP-1, which are involved in a variety of cellular inflammatory mechanisms, are modulated by macrolides [4]. Studies with LPS-primed THP-1 and human peripheral blood leukocytes in vitro have shown that erythromycin, clarithromycin and roxithromycin have an inhibitory effect on the ROS intermediate-induced activation of NF-кB. This effect seems to be dependent on cyclic AMP [59].

\subsubsection{Quinolones/Fluoroquinolones}

Quinolones have direct antibacterial effects through their inhibition of gyrase and topoisomerase IV - enzymes needed for bacterial replication. These enzymes do not exist in eukaryotic cells, but the accumulation of quinolones in mammalian cells can have an effect on the topoisomerase type II enzymes and thereby, exert harmful effects in humans when overdosed [60]. Beside these direct antibacterial effects, fluoroquinolones exhibit several immunomodulatory effects. They influence phagocytic function, $\mathrm{T}$ cell response, cytokine production and transepithelial chlorine secretion $[35,61]$. In vitro, ciprofloxacin and moxifloxacin cause a dose-dependent reduction of IL-4 and IFN- $\gamma$ generation by T cells [62]. Furthermore, moxifloxacin inhibits IL- $1 \alpha$ and TNF- $\alpha$ secretion from LPS-stimulated human monocytes at therapeutic concentrations [63]. Additionally, moxifloxacin suppresses ERK1/2 activation as well as NF- $\mathrm{KB}$ translocation in a lung respiratory cell line and in human monocytes $[64,65]$. Especially moxifloxacin seems to be an agent for the treatment of chronic airway inflammatory diseases since it also inhibits MAPK activation and production of pro-inflammatory cytokines more efficiently than ciprofloxacin or the macrolide azithromycin [66]. Trovafloxacin decreases the production of cytokines (IL- $1 \alpha$, IL-1 $\beta$, IL-6, IL-10, GM-CSF, TNF$\alpha$ ) in LPS-stimulated monocytes [67]. This immunosuppressive effect is 
regulated via the formation of prostaglandin $E_{2}$ which leads to an increase in intracellular cAMP - a key factor for immunosuppressive effects in monocytes. In LPS-stimulated monocytes, pefloxacin and ciprofloxacin reduce the production of IL-1 at doses below $100 \mathrm{mg} \mathrm{L}^{-1}$. Ofloxacin and ciprofloxacin reduce the synthesis of TNF- $\alpha$ at concentrations below $25 \mathrm{mg} \mathrm{L}^{-1}$. Grapafloxacin inhibits IL-8 production in TNF- $\alpha$ stimulated cells [68]. In vivo, ciprofloxacin and trovafloxacin were able to rescue mice injected with a lethal dose of LPS by decreasing serum IL-6 and TNF- $\alpha$ levels [69]. Moxifloxacin reduced IL- 8 and TNF- $\alpha$ levels and protected immunosuppressed mice from neutrophilic pneumonitis [70]. Taken together, fluoroquinolones reveal antiinflammatory and immunosuppressive effects indicating a potential beneficial effect in the induction of inflammatory resolution.

\subsubsection{Fosfomycin}

Fosfomycin has a special place among the antibacterials since it is structurally unrelated to any other antibacterial compound. Similar to macrolides, fosfomycin is able to reduce cytokine production by inhibition of NF- $\mathrm{KB}$ activation [71,72]. After LPS injection into mice, fosfomycin decreased peak serum levels of IL-1 $\beta$ and TNF- $\alpha$. Furthermore, local PGE 2 and TNF- $\alpha$ concentrations, as well as cyclooxygenase- 2 mRNA were reduced in the rat carrageenan air-pouch model. $\mathrm{T}$ and $\mathrm{B}$ cells were also modulated by fosfomycin and histamine release from basophils was inhibited [35]. Taken together, fosfomycin has immunomodulatory activity which could also be demonstrated in multiple animal models and in clinical trials with severe bronchial asthma patient $[35,73]$.

\subsubsection{Cyclines/Tetracyclines}

Cyclines have direct antibacterial effects through the inhibition of bacterial protein synthesis, as well as immunomodulatory functions via inhibition of different phagocyte functions like cytokine release at clinical relevant concentrations [35]. Beside their antibacterial effects, cyclines have antiinflammatory and bone resorption-inhibiting effects. Minocycline has been the target of multiple studies in arthritis models and provides a modest improvement in the course of the disease [74]. The anti-inflammatory properties of cyclines are based on the downregulation of the metalloproteinases MMP-2 and MMP-3. Tetracyclines are able to block these enzymes by chelating metal ions and without the cofactor $\mathrm{ZN}^{2+}$, the enzyme activity of the metalloproteinase is decreased $[75,76]$. This effect has been utilized in the treatment of rheumatoid arthritis and in infectious diseases. Numerous beneficial clinical effects of cyclines are based on this protease inhibition. The dimethylamino group at the position $\mathrm{C} 4$ of the tetracyclines is essential for the antibacterial activity since removal of this functional group leads to abolition of the antibacterial activity. Only one derivative of this type, CMT-3, retains inhibitory activity against metalloproteinases [77]. Besides 
blocking metalloproteinases, tetracyclines show further immunomodulatory effects like inhibition of T cell activity and of pro-inflammatory cytokine levels (IL-2, TNF, IL-1) [78,79]. Tetracyclines are also able to act as ROS scavengers thereby, ameliorating the inflammatory state by decreasing tissue damage caused by ROS [24]. Nevertheless, the potential use of tetracyclines in the treatment of chronic inflammatory diseases and bacterial infections has not been fully exploited because of possible side effects and the danger of bacterial resistance.

\subsubsection{Dapsone}

Dapsone has antiprotozoal as well as antibacterial effects and is widely used for the therapy of leprosy - usually in combination with rifampicin [80]. Additionally to direct antimicrobial effects, dapsone shows various immunomodulatory effects like inhibition of ROS generation by neutrophils, competitive inhibition of myeloperoxidase (leading to reduction of proinflammatory hypochlorous acid), inhibition of prostaglandin synthesis and inhibition of neutrophil chemotaxis [24]. Some authors have reported inhibitory effects on cytokine production - but mainly at supratherapeutic concentrations [24]. However, recently an inhibitory effect of dapsone was observed on IL-8 mRNA expression and release in human bronchial epithelial cells in vitro at low concentrations comparable to those in the serum of treated patients. In vivo, dapsone also inhibited neutrophil infiltration into the trachea of ferrets treated intratracheally with bacterial LPS [81]. IL-8 plays a major role in skin inflammation and the inhibitory effect of dapsone of IL-8 generation might explain the beneficial effect of dapsone in models of experimentally induced erythema [24]. In clinical practice, dapsone is a first choice drug for the therapy of chronic inflammatory skin diseases with neutrophilic or eosinophilic cell infiltration (e.g. dermatitis herpetiformis, IgA pemphigus, prurigo pigmentosa) [80]. Furthermore, dapsone has been tested for the therapy of rheumatoid arthritis, immune thrombocytopenia and asthma, but no recommendations have been made for these indications [24]. The anti-inflammatory properties of dapsone could be used potentially to reduce the exaggerated inflammation seen during bacterial induced diseases.

\subsubsection{6. $\beta$-Lactams}

One of the first antibiotic substance used in modern medicine was penicillin followed by its derivatives. They act via inhibition of bacterial peptidoglycan synthesis, resulting in bacterial cell lysis. These $\beta$-lactam antibiotics are facing bacterial resistance mechanisms mainly as a result of produced $\beta$-lactamase. Beside their direct antimicrobial effects, $\beta$-lactams inhibit platelet function, undergo dysulfuric reactions as well as causing osmotic diuresis. Furthermore, they inhibit IFN- $\gamma$ production, but the different $\beta$-lactams show variability in potency. Through inhibition of IFN- $\gamma$ production, $\beta$-lactams modulate IL-4 production and IgE synthesis by Th2 T cells $[82,83]$. IL-1 and TNF- $\alpha$ might also 
be influenced by the changes in IFN- $\gamma$ levels, but other cytokines are not affected by $\beta$-lactams in general [84]. Some individual $\beta$-lactams have a unique impact on the immune system. Thus, ceftriaxone interacts with the glutamate transporter-1 which is associated with several neurological diseases like stroke, epilepsy and amyotrophic lateral sclerosis. Ceftriaxone has protective effects on neurons and muscles by enhancing the transporter activity [85]. Cefaclor promotes phagocytosis by inducing a pro-inflammatory response of type 1 and increased chemotaxis [86]. Hence, even as far as one of the oldest class of antibacterial agents is concerned, immunomodulatory properties of the compounds are observed.

\subsubsection{Effects on inflammatory resolution}

Beside their effects on the initiation and progress of the inflammatory response, antibiotics are also able to facilitate the resolution of inflammation through modulation of leucocyte apoptosis. The targeted induction of apoptosis is a promising new approach to the therapy of chronic inflammatory disease and complementary to antibacterial treatment, since it facilitates clearance of damaged tissue $[87,88]$. Mainly neutrophils are addressed for this purpose since they play a major role in acute inflammation and their apoptosis is a well-regulated process. In the course of host defense, apoptosis is induced in neutrophils by phagocytosis of invading bacteria. Apoptosis promotes specific gene-mediated attenuation of several functional aspects of neutrophils [7]. Apoptotic, but not necrotic, neutrophils are ingested by macrophages [89]. Several macrolides influence the apoptosis of neutrophils and therefore, regulate the clearance mechanisms at the site of inflammation. Some studies showed a pro-apoptotic effect of erythromycin, which is at least partially cAMP-dependent [90]. This mechanism was observed for erythromycin and roxithromycin in isolated human neutrophils and guinea-pig eosinophils stimulated by IL-5 [91,92]. Azithromycin is also able to induce apoptosis in neutrophils without inducing the oxidative burst or pro-inflammatory IL-8 release. Interestingly, this effect was prevented in the presence of $S$. pneumonia [93], which might be due to the already high levels of apoptosis in the neutrophils after contact with the bacteria [7]. In a whole blood model, proapoptotic properties of erythromycin and azithromycin were shown by flow cytometry [94]. Tilmicosin, a macrolide which is used in veterinary medicine, induced apoptosis in isolated peripheral neutrophils after $2 \mathrm{~h}$ incubation [95]. Interestingly, in contrast to azithromycin, the effects of tilmicosin on apoptosis were not affected by the presence of Pasteurella haemolytica. Either the particular type of bacteria influences the apoptosis of neutrophils in diverse ways or the individual macrolides have unique pro-apoptotic effects. A 17membered tylosine derivative has pro-apoptotic effects in different cell lines [96] while the 16-membered macrolide josamycin has no effect on human neutrophil apoptosis [92]. The modulation of the NF- $\kappa$ B pathway by antibiotics is also controversial. In vitro, inhibition of NF- $\mathrm{KB}$ stimulates apoptosis in 
granulocytes [87], but an in vivo study showed a more complex mechanism. Thus, during the onset of inflammation, activation of NF- $\mathrm{KB}$ leads to proinflammatory gene expression while activation during the resolution of inflammation results in anti-inflammatory gene expression and apoptosis [97]. In a study with human volunteers, administration of azithromycin for three days caused initial stimulation of neutrophil degranulation and subsequent prolonged inhibition. Isolated circulating neutrophils showed detectable levels of azithromycin and increased apoptosis for up to 28 days after the last treatment [46]. Taken together, these studies suggest that the effects of macrolides on apoptosis are time-dependent and associated with the status of NF- $\mathrm{KB}$. A more recent study showed that the treatment of human alveolar macrophages in vitro with erythromycin, clarithromycin and azithromycin (14- and 15-membered macrolides) stimulated the phagocytosis of apoptotic neutrophils by macrophages [98]. 16-Membered macrolides like clindamycin were not studied. Additionally, tilmicosin seems to promote macrophage-mediated phagocytosis of neutrophils [95]. Consistent with the other immunomodulating effects of macrolides, the 14- and 15-membered (but not the 16-membered) macrolides are thus, able to increase inflammatory resolution by directly enhancing neutrophil apoptosis and their subsequent phagocytosis by macrophage. Macrolides are not the only antibiotics which influence the mechanisms of apoptosis, however, in many cases apoptosis is delayed or even inhibited. Minocycline inhibits apoptosis in experimental neuroinflammatory disorders [99] and rifampicin inhibits antiCD95-mediated apoptosis in peripheral blood lymphocytes and Jurkat $\mathrm{T}$ cells. The mechanism partially involves glucocorticoid receptor activation and the signaling pathway of NF- $\kappa B$ [100,101]. Furthermore, tosufloxacin, as the only quinolone antibiotic, was reported to delay neutrophil apoptosis in vitro. The delay could be reduced by a p38 mitogen-activated protein kinase (MAPK) inhibitor [102]. Taken together, the induction of apoptosis is an essential step in the inflammatory resolution which can be facilitated mainly by macrolides but also other antibacterials.

\subsection{CONCLUSION}

The modulation of the inflammatory response and the enhancement of host defense is a complex process which can be affected by a broad range of different antibiotics. Macrolides, quinolones and cyclones have the greatest impact. In the early stages of inflammation, macrolides exert inhibitory effects on the adhesion and the subsequent transepithelial migration of leukocytes. Clofazimine might have similar effects in this phase. Leukocytes can be stimulated by these antibacterials as well as by the quinolone moxifloxacin. The stimulation of host defense enhances bacterial killing in addition to the 
direct antibacterial effects of the antibiotics and is independent of potential bacterial resistance. The reduction of leukocyte migration and other inflammatory responses by macrolides as well as quinolones leads to a reduction of the inflammation process in vivo. Several antibiotics show ameliorating effects on pro-inflammatory cytokine release which might allow the use of antibiotics in anti-inflammatory approaches as with macrolides in the treatment of DPB [17]. The observation that cyclines are able to inhibit metalloproteinase release and reduce connective tissue breakdown offers several interesting approaches besides their direct antibacterial actions, as in the use of minocycline in periodontal and rheumatic diseases. Not only is the initiation of inflammation influenced by several antibiotics, also the resolution of the inflammatory status is promoted. Thus, macrolides stimulate apoptosis in leukocytes which prevents unwanted tissue destruction subsequent to ongoing inflammation. Their additional effects, like reduction of mucus secretion, enables macrolides to be used in the treatment of respiratory diseases. Beside macrolides, quinolones and cyclines are promising agents for new approaches in the treatment of inflammatory conditions. Sulphasalazine is an example of the use of an antibiotic as anti-inflammatory drug. This transfer from "the classical use" of antibiotics is a promising approach towards the treatment of chronic inflammatory diseases. For the treatment of bacterial infections, the early enhancement of host defense mechanisms is important to reduce the invading pathogens. The time dependent enhancement of apoptosis of the immune cells helps to facilitate the resolution of the inflammatory status to prevent unwanted tissue damage through prolonged inflammation. The major advantage of immunomodulatory antibiotics is that this additional activity is seen even in the presence of bacterial resistance as bacterial killing occurs as a result of enhanced host defense mechanisms and not just through the direct antibacterial properties of the antibiotics.

\section{ACKNOWLEDGEMENT}

We thank the LOEWE Research Centre for Translational Medicine and Pharmacology of the State of Hessen for financial support. 


\section{REFERENCES}

1. M.J. Parnham, Infektol. glasn./Croat. J. Infect. 31 (2011) 15-27.

2. J.Y. Min, Y.J. Jang, Mediators Inflamm. 2012 (2012) Article ID 649570.

3. M.J. Parnham, Curr Opin Infect Dis. 18 (2005) 125-131.

4. $\quad$ O. Culic, V. Erakovic, M.J. Parnham, Eur. J. Pharmacol. 429 (2001) 209-229.

5. C.T. Robb, K.H. Regan, D.A. Dorward, A.G. Rossi, Semin. Immunopathol. 38 (2016) 425-448.

6. D.W. Gilroy, P.R. Colville-Nash, S. McMaster, D.A. Sawatzky, D.A. Willoughby, T. Lawrence, FASEB J. 17 (2003) 2269-2271.

7. S.D. Kobayashi, J.M. Voyich, F.R. DeLeo, Microbes Infect. 5 (2003) 1337-1344.

8. J. Savill, Kidney Blood Press Res. 23 (2000) 173-174.

9. M. Bosnar, Z. Kelneric, V. Munic, V. Erakovic, M.J. Parnham, Antimicrob. Agents Chemother. 49 (2005) 2372-2377.

10. M.T. Labro, in Macrolide Antibiotics, Milestones in Drug Therapy MDT, W. Schönfeld, H.A. Kirst (Eds.), Birkhäuser Verlag, Basel, Switzerland, 2002, p. 3752.

11. L.E. Bermudez, C. Inderlied, L.S. Young, Antimicrob. Agents Chemother. $\mathbf{3 5}$ (1991) 2625-2629.

12. R.P. Gladue, G.M. Bright, R.E. Isaacson, M.F. Newborg, Antimicrob. Agents Chemother. 33 (1989) 277-282.

13. A. Fietta, C. Merlini, G. Gialdroni Grassi, J. Chemother. 9 (1997) 23-31.

14. W.L. Hand, R.W. Corwin, T.H. Steinberg, G.D. Grossman, Am. Rev. Respir. Dis. 129 (1984) 933-937.

15. C.S. Easmon, J.P. Crane, J. Antimicrob. Chemother. 16 (1985) 67-73.

16. W.A. Muller, Trends Immunol. 24 (2003) 327-334.

17. M.J. Parnham, in Antibiotics as Anti-Inflammatory and Immunomodulatory Agents, B.K. Rubin, J. Tamaoki (Eds.), Birkhäuser Verlag, Basel, Switzerland, 2005, p. 27-47.

18. S. Kusano, J. Kadota, S. Kohno, K. Iida, K. Kawakami, T. Morikawa, K. Hara, Respiration. 62 (1995) 217-222.

19. F. Enomoto, G. Ichikawa, I. Nagaoka, T. Yamashita, Nihon Jibiinkoka Gakkai Kaiho. 99 (1996) 1126-1135.

20. F. Enomoto, G. Ichikawa, I. Nagaoka, T. Yamashita, Acta Otolaryngol. Suppl. 539 (1998) 57-60.

21. S. Kawasaki, H. Takizawa, T. Ohtoshi, N. Takeuchi, T. Kohyama, H. Nakamura, T. Kasama, K. Kobayashi, K. Nakahara, Y. Morita, K. Yamamoto, Antimicrob. Agents Chemother. 42 (1998) 1499-1502.

22. O.A. Khair, J.L. Devalia, M.M. Abdelaziz, R.J. Sapsford, R.J. Davies, Eur. Respir. J. 8 (1995) 1451-1457.

23. N. Matsuoka, K. Eguchi, A. Kawakami, M. Tsuboi, Y. Kawabe, T. Aoyagi, S. Nagataki, Clin. Exp. Immunol. 104 (1996) 501-508.

24. M.J. Parnham, V. Erakovic Haber, in Compendium of Inflammatory Diseases, M.J. Parnham (Ed.), Springer, Basel, Switzerland, 2016, p. 77-86.

25. T. Mitsuyama, K. Hidaka, T. Furuno, N. Hara, Int. Arch. Allergy Immunol. 114 (1997) 111-115.

26. J. Tamaoki, M. Kondo, K. Kohri, K. Aoshiba, E. Tagaya, A. Nagai, J. Immunol. 163 (1999) 2909-2915. 
27. L. Baranda, B. Torres-Alvarez, R. Cortes-Franco, B. Moncada, D.P. PortalesPerez, R. Gonzalez-Amaro, Arch Dermatol. 133 (1997) 325-329.

28. J. Kadota, O. Sakito, S. Kohno, H. Sawa, H. Mukae, H. Oda, K. Kawakami, K. Fukushima, K. Hiratani, K. Hara, Am. Rev. Respir. Dis. 147 (1993) 153-159.

29. H. Oda, J. Kadota, S. Kohno, K. Hara, Chest. 106 (1994) 1116-11123.

30. M. Kawashima, J. yatsunami, Y. Fukuno, M. Nagata, M. Tominaga, S. Hayashi, Lung. 180 (2002) 73-89.

31. A. Azuma, T. Furuta, T. Enomoto, Y. Hashimoto, K. Uematsu, N. Nukariya, A. Murata, S. Kudoh, Thorax. 53 (1998) 186-189.

32. D. Torre, M. Broggini, V. Botta, C. Sampietro, R. Busarello, C. Garberi, J. Chemother. 3 (1991) 236-239.

33. A. Ianaro, A. Ialenti, P. Maffia, L. Sautebin, L. Rombola, R. Carnuccio, T. Iuvone, F. D'Acquisto, M. Di Rosa, J. Pharmacol. Exp. Ther. 292 (2000) 156-163.

34. J. Tamaoki, Chest. 125 (2004) 41-50.

35. M.T. Labro, Clin. Microbiol. Rev. 13 (2000) 615-650.

36. C. Van Rensburg, E. Gatner, F. Inkamp, R. Anderson, Antimicrob. Agents Chemother. 21 (1982) 693-698.

37. F. Scaglione, G. Rossoni, J. Antimicrob. Chemother. 41 Suppl B (1998) 47-50.

38. J. Tamaoki, N. Sakai, E. Tagaya, K. Konno, Antimicrob. Agents Chemother. 38 (1994) 1641-1643.

39. J. Tamaoki, K. Takeyama, I. Yamawaki, M. Kondo, K. Konno, Am. J. Physiol. 272 (1997) L15-L19.

40. G. Xu, J. Fujita, K. Negayama, K. Yuube, S. Hojo, Y. Yamaji, K. Kawanishi, J. Takahara, Microbiol. Immunol. 40 (1996) 473-479.

41. E. Kita, M. Sawaki, F. Nishikawa, K. Mikasa, Y. Yagyu, S. Takeuchi, K. Yasui, N. Narita, S. Kashiba, Pharmacology 41 (1990) 177-183.

42. E. Kita, M. Sawaki, K. Mikasa, K. Hamada, S. Takeuchi, K. Maeda, N. Narita, J. Antimicrob. Chemother. 32 (1993) 285-294.

43. Y. Sugiura, O. Yoshihiro, N. Yoshiaki, Acta Oto-Laryngologica. 117(531) (1997) 39-51.

44. K. Riesbeck, J. Chemother. 14 (2002) 3-12.

45. I.H. Hall, U.E. Schwab, E.S. Ward, T.J. Ives, Life Sci. 73 (2003) 2675-2685.

46. O. Culic, V. Erakovic, I. Cepelak, K. Barisic, K. Brajsa, Z. Ferencic, R. Galovic, I. Glojnaric, Z. Manojlovic, V. Munic, R. Novak-Mircetic, V. Pavicic-Beljak, M. Sucic, M. Veljaca, T. Zanic-Grubisic, M.J. Parnham, Eur. J. Pharmacol. 450 (2002) 277289.

47. M.J. Parnham, V. Erakovic Haber, E.J. Giamarellos-Bourboulis, G. Perletti, G.M. Verleden, R. Vos, Pharmacol. Ther. 143 (2014) 225-245.

48. H.C. Steel, A.J. Theron, R. Cockeran, R. Anderson, C. Feldman, Mediators Inflamm. 2012 (2012) 584262.

49. S. Kanoh, B.K. Rubin, Clin. Microbiol. Rev. 23 (2010) 590-615.

50. C.G. Cochrane, Am. J. Med. 91 (1991) 23S-30S.

51. M.T. Labro, J. el Benna, C. Babin-Chevaye, J. Antimicrob. Chemother. 24 (1989) 561-572.

52. R. Anderson, A.J. Theron, C. Feldman. Inflammation 20 (1996) 693-705.

53. H. Terao, K. Asano, K. Kanai, Y. Kyo, S. Watanabe, T. Hisamitsu, H. Suzaki, Mediators Inflamm. 12 (2003) 195-202.

54. F. Pellacini, D. Botta, S. Romagnano, E. Moriggi, L. Pradella, US Patent No. 6,455,576. (2002) 
55. K. Mikasa, E. Kita, M. Sawaki, M. Kunimatsu, K. Hamada, M. Konishi, S. Kashiba, N. Narita, J. Antimicrob. Chemother. 30 (1992) 339-348.

56. C. Agen, R. Danesi, C. Blandizzi, M. Costa, B. Stacchini, P. Favini, M. Del Tacca, Agents Actions. 38 (1993) 85-90.

57. H. Suzaki, K. Asano, S. Ohki, K. Kanai, T. Mizutani, T. Hisamitsu, Mediators Inflamm. 8 (1999) 199-204.

58. O. Carevic, S. Djokic, Agents Actions. 25 (1988) 124-131.

59. K. Abeyama, K. Kawahara, S. Iino, T. Hamada, S. Arimura, K. Matsushita, T. Nakajima, I. Maruyama, J. Leukoc. Biol. 74 (2003) 908-915.

60. C. Sissi, M. Palumbo, Curr. Med. Chem. Anticancer Agents 3 (2003) 439-450.

61. S. Kanoh, J. Tamaoki, M. Kondo, Y. Nagano, A. Nagai, Antimicrob. Agents Chemother. 45 (2001) 2928-2930.

62. A.C. Williams, H.F. Galley, A.M. Watt, N.R. Webster, J. Antimicrob. Chemother. 56 (2005) 502-506.

63. F.G. Araujo, T.L. Slifer, J.S. Remington. Clin. Microbiol. Infect. 8 (2002) 26-30.

64. T. Weiss, I. Shalit, H. Blau, S. Werber, D. Halperin, A. Levitov, I. Fabian, Antimicrob. Agents Chemother. 48 (2004) 1974-1982.

65. S. Werber, I. Shalit, I. Fabian, G. Steuer, T. Weiss, H. Blau, J. Antimicrob. Chemother. 55 (2005) 293-300.

66. G.S. Zimmermann, C. Neurohr, H. Villena-Hermoza, R. Hatz, J. Behr, Respir. Res. 10 (2009) 89.

67. A.A. Khan, T.R. Slifer, J.S. Remington, Antimicrob. Agents Chemother. 42 (1998) 1713-1717.

68. B. Kwiatkowska, M. Maslinska, M. Przygodzka, J. Dmowska-Chalaba, J. Dabrowska, K. Sikorska-Siudek, Adv. Biosci. Biotechnol. 4 (2013) 91-101.

69. A.A. Khan, T.R. Slifer, F.G. Araujo, Y. Suzuki, J.S. Remington, Antimicrob. Agents Chemother. 44 (2000) 3169-3173.

70. I. Shalit, L. Horev-Azaria, I. Fabian, H. Blau, N. Kariv, I. Shechtman, H. Alteraz, Y. Kletter, Antimicrob. Agents Chemother. 46 (2002) 2442-2449.

71. J. Honda, Y. Okubo, M. Kusaba, M. Kumagai, N. Saruwatari, K. Oizumi, Immunopharmacology 39 (1998) 149-155.

72. Y. Yoneshima, T. Ichiyama, H. Ayukawa, T. Matsubara, S. Furukawa, Int. J. Antimicrob. Agents 21 (2003) 589-592.

73. M.T. Labro. Curr. Opin. Investig. Drugs 3 (2002) 61-68.

74. M. Stone, P.R. Fortin, C. Pacheco-Tena, R.D. Inman, J. Rheumatol. 30 (2003) 2112-2122.

75. A.N. Sapadin, R. Fleischmajer, J. Am. Acad. Dermatol. 54 (2006) 258-265.

76. T.R. Pasquale, J.S. Tan, Clin. Infect. Dis. 40 (2005) 127-135.

77. G.F. Nieman, B.R. Zerler, Curr. Med. Chem. 8 (2001) 317-325.

78. K. Guz, G. Bugla-Ploskonska, Postepy Hig. Med. Dosw. (Online). 61 (2007) 828837.

79. M. Kloppenburg, C.L. Verweij, A.M. Miltenburg, A.J. Verhoeven, M.R. Daha, B.A. Dijkmans, F.C. Breedveld, Clin. Exp. Immunol. 102 (1995) 635-641.

80. G. Wozel, C. Blasum, Arch. Dermatol. Res. 306 (2014) 103-124.

81. S. Kanoh, T. Tanabe, B.K. Rubin, Chest. 140 (2011) 980-990.

82. D.N. Wilson, Crit. Rev. Biochem. Mol. Biol. 44 (2009) 393-433.

83. M.N. Alekshun, Expert Opin. Investig. Drugs 14 (2005) 117-134.

84. B.M. Brooks, B.F. Flanagan, A.L. Thomas, J.W. Coleman, Biochem. Biophys. Res. Commun. 288 (2001) 1175-1181. 
85. S.C. Tauber, R. Nau, Curr. Mol. Pharmacol. 1 (2008) 68-79.

86. K. Mangano, C. Quattrocchi, C. Aiello, G. Scalia, A. Speciale, G. Nicoletti, R. Di Marco, J. Chemother. 18 (2006) 641-647.

87. C. Ward, I. Dransfield, E.R. Chilvers, C. Haslett, A.G. Rossi, Trends Pharmacol Sci. 20 (1999) 503-509.

88. E.R. Chilvers, A.G. Rossi, J. Murray, C. Haslett, Thorax. 53 (1998) 533-534.

89. J. Savill, C. Haslett, in Apoptosis and inflammation, J.D. Winkler (Ed.), Birkhäuser Verlag, Basel, Switzerland, 1999, p. 53-84.

90. K. Aoshiba, A. Nagai, K. Konno, Antimicrob. Agents Chemother 39 (1995) 872877.

91. T. Adachi, S. Motojima, A. Hirata, T. Fukuda, N. Kihara, A. Kosaku, H. Ohtake, S. Makino, J. Allergy Clin. Immunol. 98 (1996) S207-S215.

92. K. Inamura, N. Ohta, S. Fukase, N. Kasajima, M. Aoyagi, Rhinology. 38 (2000) 124-129.

93. C.C. Koch, D.J. Esteban, A.C. Chin, M.E. Olson, R.R. Read, H. Ceri, D.W. Morck, A.G. Buret, J. Antimicrob. Chemother. 46 (2000) 19-26.

94. D.P. Healy, P.A. Silverman, A.N. Neely, I.A. Holder, G.E. Babcock, Pharmacotherapy 22 (2002) 578-585.

95. A.C. Chin, W.D. Lee, K.A. Murrin, D.W. Morck, J.K. Merrill, P. Dick, A.G. Buret, Antimicrob. Agents Chemother. 44 (2000) 2465-2470.

96. M. Grdisa, N. Lopotar, K. Pavelic, Chemotherapy 44 (1998) 331-336.

97. T. Lawrence, D.W. Gilroy, P.R. Colville-Nash, D.A. Willoughby, Nat. Med. 7 (2001) 1291-1297.

98. T. Yamaryo, K. Oishi, H. Yoshimine, Y. Tsuchihashi, K. Matsushima, T. Nagatake, Antimicrob. Agents Chemother. 47 (2003) 48-53.

99. T. Tikka, T. Usenius, M. Tenhunen, R. Keinanen, J. Koistinaho, J. Neurochem. 78 (2001) 1409-1414.

100. R. Yerramasetti, S. Gollapudi, S. Gupta, J. Clin. Immunol. 22 (2002) 37-47.

101. S. Gollapudi, S. Jaidka, S. Gupta, J. Clin. Immunol. 23 (2003) 11-22.

102. Y. Azuma, K. Ohura, Inflammation. 27 (2003) 115-122.

(C)2018 by the authors; licensee IAPC, Zagreb, Croatia. This chapter is an open-access publication distributed under the terms and conditions of the Creative Commons Attribution license (http://creativecommons.org/licenses/by/3.0/) (cc) EY 